\title{
Tritium Specific Adsorption Simulation Utilizing the OSPREY Model
}

\author{
Veronica Rutledge \\ Lawrence Tavlarides \\ Ronghong Lin \\ Austin Ladshaw
}

September 2013

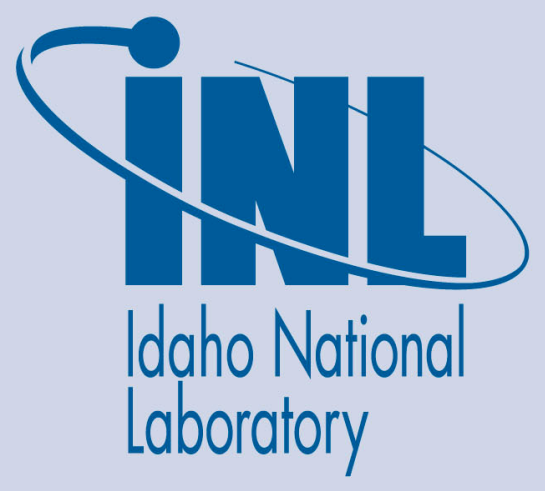

The INL is a U.S. Department of Energy National Laboratory operated by Battelle Energy Alliance 
INL/EXT-13-30274

FCR\&D-SWF-2013-000384

\title{
Tritium Specific Adsorption Simulation Utilizing the OSPREY Model
}

\author{
Veronica Rutledge \\ Lawrence Tavlarides ${ }^{1}$ \\ Ronghong Lin ${ }^{1}$ \\ Austin Ladshaw ${ }^{2}$ \\ ${ }^{1}$ Syracuse University \\ ${ }^{2}$ Georgia Institute of Technology
}

September 2013

Idaho National Laboratory

Fuel Cycle Research \& Development

Idaho Falls, Idaho 83415

http://www.inl.gov

Prepared for the

U.S. Department of Energy

Office of Nuclear Energy

Under DOE Idaho Operations Office

Contract DE-AC07-05ID14517 


\section{DISCLAIMER}

This information was prepared as an account of work sponsored by an agency of the U.S. Government. Neither the U.S. Government nor any agency thereof, nor any of their employees, makes any warranty, expressed or implied, or assumes any legal liability or responsibility for the accuracy, completeness, or usefulness, of any information, apparatus, product, or process disclosed, or represents that its use would not infringe privately owned rights. References herein to any specific commercial product, process, or service by trade name, trade mark, manufacturer, or otherwise, does not necessarily constitute or imply its endorsement, recommendation, or favoring by the U.S. Government or any agency thereof. The views and opinions of authors expressed herein do not necessarily state or reflect those of the U.S. Government or any agency thereof. 



\section{SUMMARY}

During the processing of used nuclear fuel, volatile radionuclides will be discharged to the atmosphere if no recovery processes are in place to limit their release. The volatile radionuclides of concern are ${ }^{3} \mathrm{H},{ }^{14} \mathrm{C},{ }^{85} \mathrm{Kr}$, and ${ }^{129} \mathrm{I}$. Methods are being developed, via adsorption and absorption unit operations, to capture these radionuclides. It is necessary to model these unit operations to aid in the evaluation of technologies and in the future development of an advanced used nuclear fuel processing plant. A collaboration between Fuel Cycle Research and Development Offgas Sigma Team member INL and a NEUP grant including ORNL, Syracuse University, and Georgia Institute of Technology has been formed to develop off gas models and support off gas research. This report is discusses the development of a tritium specific adsorption model. Using the OSPREY model and integrating it with a fundamental level isotherm model developed under and experimental data provided by the NEUP grant, the tritium specific adsorption model was developed. 


\section{CONTENTS}

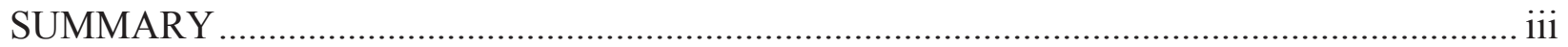

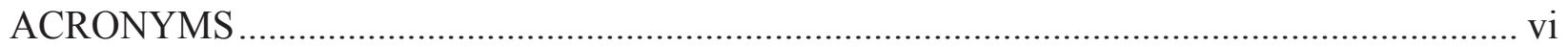

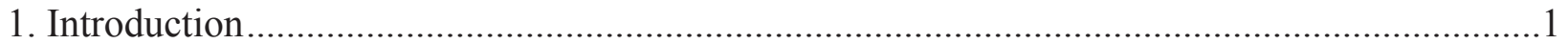

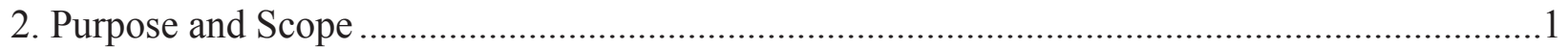

3. Tritium Specific Adsorption Model ................................................................................ 1

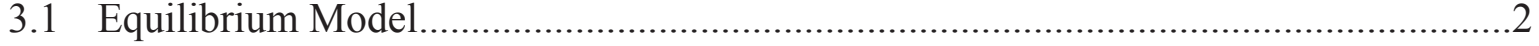

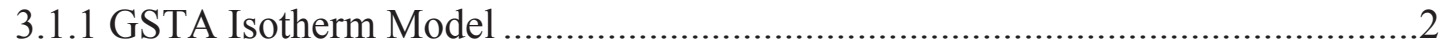

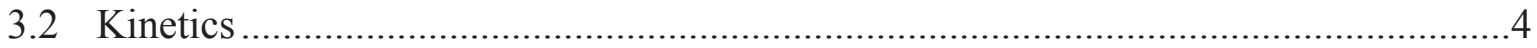

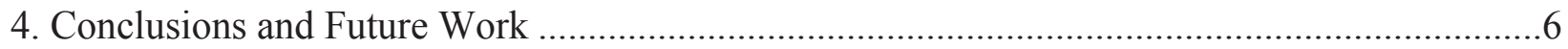

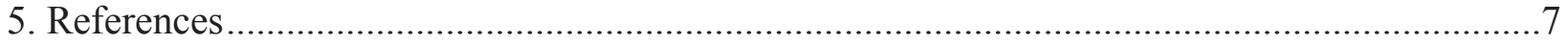




\section{FIGURES}

Figure 1: Krypton adsorption at $191 \mathrm{~K}$ on HZ PAN. Comparison of using Langmuir Isotherm model vs. GSTA Isotherm model with $\mathrm{m}=1$ for verification that GSTA isotherm model works when integrated with OSPREY model. 


\section{ACRONYMS}

HZ-PAN Hydrogen engineered form sorbent

INL Idaho National Laboratory

K Kelvin $\left({ }^{\circ} \mathrm{C}+273.15\right)$

$\mathrm{Kr} \quad$ krypton

UNF Used Nuclear Fuel

ORNL Oak Ridge National Laboratory

SU Syracuse University

GIT Georgia Institute of Technology

MOOSE Multi Object Oriented Simulation Environment

OSPREY Offgas SeParation and RecoverY

GSTA Generalized Statistcal Thermodynamic Adsorption

LDF Linear Driving Force

SC Shrinking Core

NEUP Nuclear Energy University Program 



\section{Tritium Specific Adsorption Simulation Utilizing the OSPREY Model}

\section{Introduction}

The reporocessing of used nuclear fuel (UNF) generates off-gas containing several radioactive gases including ${ }^{129} \mathrm{I}_{2},{ }^{85} \mathrm{Kr},{ }^{3} \mathrm{H}_{2} \mathrm{O}$, and ${ }^{14} \mathrm{CO}_{2}$. The unit operations of adsorption and absorption are being developed for the removal of these off-gas constituents. It is necessary to model complex series of unit operations simulating the off gas treatment system of a UNF reprocessing facility. These simulations will aid in the future design of advanced U.S. reprocessing plants as well as minimize waste, proliferation risk, environmental impact, process complexity and cost.

In support of the DOE Fuel Cycle Research and Development Offgas Sigma Team and a Nuclear Energy University Program (NEUP) grant including Oak Ridge National Laboratory (ORNL), Syracuse University (SU), and Georgia Institute of Technology (GIT), a tritium specific model has been developed within the MOOSE application OSPREY for the adsorption of water vapor on molecular sieve 3A. The delivery of this report satisfies milestones M4FT13IN03120212 and M3FT-13IN03120211.

\section{Purpose and Scope}

The purpose of this work was to update and maintain OSPREY and to add a tritium specific model to the OSPREY application while improving OSPREY as more $\mathrm{Kr}$ data became available and integrating the Sigma Offgas Team INL modeling effort with the NEUP grant offgas work. Throughout FY-13, OSPREY has been modified to comply with changes and upgrades to MOOSE. These modifications include generating tests with tighter tolerances to verify that OSPREY remains functional while the modeling environment improves and provides a higher fidelity. Also, in support of the krypton portion of the OSPREY model, more experimental data for krypton adsorption were provided to further validate this krypton specific model; however without having experimentally determined kinetic data and parameters for krypton to complement the equilibria data, no validatation graphs are shown in this report.

Additional updates to OSPREY, which are discussed within this report, include integration with a GIT developed isotherm model and collaboration with SU for the provision of experimental data and kinetic parameters. This report does not include any tritium specific model validation graphs as a result of the dew point meter at $\mathrm{SU}$ providing incorrect readings for the data initially collected and provided to INL for model support. This error was not detected in time to correct the data and parameters provided prior to this report; however, the model will be validated early in FY-14 as updated data become available.

\section{Tritium Specific Adsorption Model}

The OSPREY model provides the necessary framework for the tritium specific adsorption model because the governing equations are generalized for adsorption and do no change based on the gas/sorbent system. A detailed description of the OSPREY model was previously reported ${ }^{1}$. Changes that were necessary due to the change of the gas/sorbent system were the isotherm model and the kinetic equations. 


\subsection{Equilibrium Model}

Due to the fact that the OSPREY model was initially generated as a krypton specific model, the equilibrium model used was the Langmuir Isotherm model. According to literature ${ }^{2}$, tritium adsorption does not fit the Langmuir Isotherm model. Therefore, to accurately model the equilibrium for the tritium specific model the Langmuir Isotherm model has been replaced with a Generalized Statistical Thermodynamic Adsorption (GSTA) Isotherm model that was created by GIT. Since this is a generalized isotherm model, it will work for a wide variety of isotherms, including the Langmuir Isotherm. Therefore, integration of OSPREY with the GIT developed isotherm model broadens the use of OSPREY beyond simply Langmuir type adsorption. OSPREY, integrated with the GSTA isotherm model will work for krypton adsorption as well as tritium adsorption.

\subsubsection{GSTA Isotherm Model}

The Generalized Statistical Thermodynamic Adsorption (GSTA) isotherm model, summarized in equations 1 through 3, was originally developed by Llano-Restrepo and Mosquera $^{2}$ as an alternative presentation of Hill's statistical isotherm model of adsorption. This recast version of Hill's model allows for a simpler physical interpretation of the parameters as follows: $q_{\max }$ represents the theoretical maximum adsorption capacity, $m$ is the number of adsorption sites (or subsystems) available to adsorbate molecules, $K_{n}$ is the equilibrium constant relative to the adsorption of $n$ molecules in the network of available adsorption sites, $K_{n}{ }^{o}$ is the dimensionless equilibrium constant which is relative to the standard state pressure $\left(P^{o}\right)$ of 100 $\mathrm{kPa}, \Delta H_{n}{ }^{o}$ is the standard molar enthalpy of the adsorption of $n$ molecules in a network of sites, and $\Delta S_{n}{ }^{\circ}$ is the standard molar entropy of adsorption of $n$ molecules in the same network of sites.

$$
\begin{gathered}
q=\frac{q_{\max }}{m} \frac{\sum_{n=1}^{m} n K_{n} p^{n}}{\mathbf{1}+\sum_{n=1}^{m} K_{n} p^{n}} \\
K_{n}=K_{n}^{o} /\left(P^{o}\right)^{n} \\
\ln K_{n}^{o}=-\frac{\Delta H_{n}^{o}}{R T}+\frac{\Delta S_{n}^{o}}{R}
\end{gathered}
$$

The dimensionless constant is related to temperature by the van't Hoff equation (equation 4). Integration of this equation with temperature results in equation 3 above. Once the parameters of the GSTA model are determined, the corresponding enthalpies and entropies are found by performing a linear regression across temperature for all $K_{n}^{o}$ values. With the determination of the appropriate enthalpies and entropies for a specific adsorbent-adsorbate system, it is then possible to predict the isotherm curves at temperatures for which data was not obtained.

$$
\left(\frac{\partial \ln K_{n}^{o}}{\partial T}\right)_{n}=\frac{\Delta H_{n}^{o}}{R T^{2}}
$$

Some notable properties of the GSTA isotherm model are linear low pressure behavior, adaptability to represent various degrees of heterogeneity of adsorbents, and flexibility to 
describe a wide variety of data sets. One of the more rigorous measures of an adsorption isotherm model is the behavior displayed at low pressures in the so called Henry Regime of adsorption. As the pressure in a system approaches zero, the isotherm is expected to display a linear relationship, equation 5, between solid and gas phases (i.e. Henry's Law). Equation 6 below shows the determination of the Henry's Law constant that results by taking the limit of the GSTA isotherm as pressure approaches zero.

$$
\begin{gathered}
q=K_{H} p \\
K_{H}=\lim _{p \rightarrow 0} \frac{q}{p}=\frac{K_{1}^{o} q_{\max }}{m P^{o}}
\end{gathered}
$$

The adaptability and flexibility of the GSTA model is immediately apparent by simply considering the $m$ parameter from equation 1 above. When $m$ is increased, the physical interpretation is that more adsorption sites are available and therefore the adsorbent is more heterogeneous. At the same time, allowing the $m$ parameter to be flexible when performing an equilibrium analysis will allow the polynomial section of the model to expand, thus resulting in tighter agreement between the model and the collected data. Under the condition in which the $m$ parameter is equal to one, then the form of the GSTA model is the exact same form of the Langmuir model, which is a homogeneous isotherm model.

Upon integration of the GSTA isotherm model with OSPREY, it was verified using the krypton specific model for the case mentioned in the previous paragraph of $m$ equal to one. Since the krypton specific model was originally generated using the Langmuir Isotherm model the results from this were compared to the results generated after replacing the Langmuir Isotherm model with the GSTA model when $\mathrm{m}$ is equal to one. This comparison is shown in Figure 1.

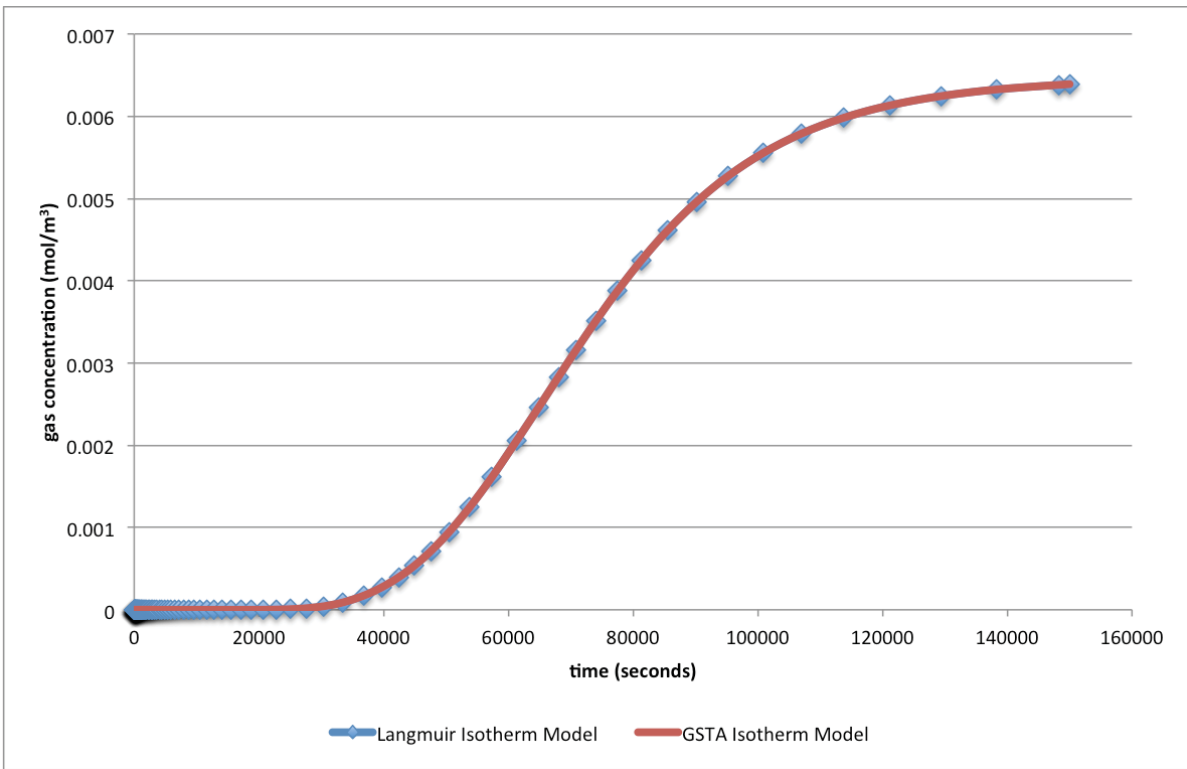

Figure 1: Krypton adsorption at $191 \mathrm{~K}$ on HZ PAN. Comparison of using Langmuir Isotherm model vs. GSTA Isotherm model with $m=1$ for verification that GSTA isotherm model works when integrated with OSPREY model. 
Figure 1 shows that, when compared, the use of the Langmuir Isotherm model and the GSTA Isotherm model with $m$ equal to one do not generate different OSPREY model output. This provides verification that when integrated with OSPREY, the GSTA Isotherm model works. For the tritium specific model, the GSTA Isotherm model parameter $m$ is greater than one in order to better fit the isotherm and determine the equilibrium parameters.

\subsection{Kinetics}

The kinetic parameters for tritium adsorption were experimentally determined by SU. SU performed water vapor adsorption testing on molecular sieve 3A. Uptake curves for water adsorption on molecular sieve $3 \mathrm{~A}$ were obtained at adsorption temperatures of 25, 40, 60 and $80^{\circ} \mathrm{C}$ and over a dew point range from $-70{ }^{\circ} \mathrm{C}$ to $10^{\circ} \mathrm{C}$. Selected uptake curves were fitted with several adsorption kinetic models including the Langmuir model, the $x^{\text {th }}$-order kinetic model, the Elovich model, the Weber-Morris model, the Double Exponential model, the Linear Driving Force (LDF) model and the Shrinking Core (SC) model $^{3-6}$. It was found that the LDF and SC models best fit the experimental data. Therefore, the LDF and SC models were used to correlate all water uptake curves and the corresponding kinetic parameters were determined. The initial tritium specific model has been generated using the LDF model for the kinetics.

\subsubsection{Linear Driving Force Model}

Originally proposed by Gleuckauf and Coates in 1947, the linear-driving force (LDF) model has been widely used in modeling adsorption kinetics due to its analytical simplicity ${ }^{7}$. According to the LDF model, the average sorbate uptake rate is proportional to the amount still required to reach equilibrium, as given by:

$$
\frac{d q}{d t}=k\left(q_{e}-q\right)
$$

where $q$ is the average sorbate concentration in sorbents, $q_{e}$ is the equilibrium sorbate concentration in sorbents and $k$ is the lumped LDF mass transfer coefficient. Integrating Equation 7 results in:

$$
\ln \frac{q_{e}-q}{q_{e}}=-k t
$$

The LDF mass transfer coefficient can be determined by plotting $\ln \frac{q_{e}-q}{q_{e}}$ versus $t$.

Assuming a linear isotherm $q=K C$, it can be shown that the LDF mass transfer resistance has the following expression [6]:

$$
\frac{1}{k}=\frac{R_{p}}{3 k_{f}} \frac{q_{e} \rho_{p}}{C_{b}}+\frac{R_{p}^{2}}{15 \varepsilon_{p} D_{p}} \frac{q_{e} \rho_{p}}{C_{b}}+\frac{R_{c}^{2}}{15 D_{c}}
$$

where, $C_{b}$ - bulk gas-phase concentration

$D_{c}$ - micropore diffusivity

$D_{p}$ - macropore diffusivity 
$k_{f}-$ film mass transfer coefficient

$R_{c}$ - radius of micropore

$R_{p}$ - radius of pellet

$\varepsilon_{p}$ - porosity of pellet

$\rho_{p}-$ density of pellet

The three terms (from left to right) in the right-hand side of Equation 9 are external film resistance, macropore resistance and micropore resistance, respectively.

The overall mass transfer resistances $(1 / k)$ were determined in the order of magnitude from $10^{-1}$ to $10^{2} \mathrm{hr}$. The micropore diffusivity $\left(D_{c}\right)$ in zeolite is usually in the order of magnitude of $10^{-}$ ${ }^{8} \mathrm{~cm}^{2} / \mathrm{s}$ (ref. 8), which leads to a micropore resistance of $\sim 4 \times 10^{-5} \mathrm{hr}^{-1}$, which is much smaller than the overall mass resistance and therefore can be neglected. Accordingly, Equation 9 can be simplified as:

$$
\frac{1}{k}=\frac{R_{p}}{3 k_{f}} \frac{q_{e} \rho_{p}}{C_{b}}+\frac{R_{p}^{2}}{15 \varepsilon_{p} D_{p}} \frac{q_{e} \rho_{p}}{C_{b}}
$$

The film mass transfer coefficient $\left(k_{f}\right)$ in Equation 10 can be estimated by the Ranz and Marshall correlation":

$$
S h=2+0.6 S c^{1 / 3} \operatorname{Re}^{0.5}
$$

where $S h, S c$ and Re are the dimensionless Sherwood number, Schmidt number and Reynolds number. It should be mentioned that the current water uptake curves were obtained in a singlelayer adsorption bed where the single layer of sorbents was sitting on a screen pan suspended on a microbalance arm. Equation 11 is for mass transfer to unobstructed spheres and we assume that the effect of the support screen used to hold the single-layer of sorbents was negligible. For correlating deep bed data, the Wakao and Funazkri correlation ${ }^{10}$ is recommended:

$$
S h=2+1.1 S c^{1 / 3} \operatorname{Re}^{0.6}
$$

The water molecular diffusivity required for the calculation can be estimated by the Fuller et al. correlation $^{11}$.

$$
D_{A B}=\frac{0.00143 T^{1.75}}{P M_{A B}^{1 / 2}\left[\left(\Sigma_{v}\right)_{A}^{1 / 3}+\left(\Sigma_{v}\right)_{B}^{1 / 3}\right]^{2}}
$$

Finally, the macropore diffusivity can be determined from Equation 10.

\subsubsection{Shrinking Core Model}

The shrinking-core model is also frequently used in modeling adsorption kinetics. This model simplifies the adsorption process in three $\operatorname{steps}^{3,8}:$ (1) diffusion through external gas film, (2) diffusion through saturated shell, and (3) adsorption at the surface of the sorbate-free core. Assuming that the third step occurs sufficiently rapidly, the sorbate uptake curve can be expressed by: 


$$
\begin{gathered}
t=\frac{q}{q_{e}} \tau_{1}+\left(1+2\left(1-\frac{q}{q_{e}}\right)-3\left(1-\frac{q}{q_{e}}\right)^{2 / 3}\right) \tau_{2} \\
\tau_{1}=\frac{R_{p} q_{e}}{3 \varepsilon_{p} k_{f} C_{b}} \\
\tau_{2}=\frac{R_{p}^{2} q_{e}}{6 \varepsilon_{p} D_{p} C_{b}}
\end{gathered}
$$

The first and second terms in the right-hand side of Equation 14 correspond to steps (1) and (2), respectively. This model has been successfully used for the analysis of water uptake curves at or near ambient temperature in molecular sieve adsorbents ${ }^{8}$.

It is reasonable to assume that the adsorption kinetics is controlled by the external film mass transfer in the very beginning of the adsorption process. Therefore, $\tau_{l}$ was determined by plotting $q / q_{e}$ versus $t$ at $q / q e<0.1$. $\tau_{2}$ was then determined by plotting $1+2\left(1-\frac{q}{q_{e}}\right)-3\left(1-\frac{q}{q_{e}}\right)^{2 / 3}$ versus $t-\frac{q}{q_{e}} \tau_{1}$ at $q / q e<0.9$. Finally, $k_{f}$ and $D_{p}$ were determined by Equations 15 and 16 , respectively.

\section{Conclusions and Future Work}

In conclusion, an initial tritium specific adsorption model has been developed within the OSPREY model. Also, a beneficial collaboration with the NEUP grant has been established and will continue along with the INL off gas modeling effort. The tritium model will be validated early in FY-14 using data generated by SU. Additional future work for the OSPREY model includes validation of multiple component models and integration with a fundamental level kinetic model developed by GIT. Kinetic data for krypton adsorption is needed to further develop and validate the krypton specific model more accurately. 


\section{References}

1. VERONICA RUTLEDGE, “OSPREY Model,” FCRD-SWF-2013-000086, January 2013.

2. M. LLANO-RESTREPO and M.A. MOSQUERO, "Accurate correlation, thermochemistry, and structural interpretation of equilibrium adsorption isotherms of water vapor in zeolite $3 \mathrm{~A}$ by means of a generalized statistical thermodynamic adsorption model," Fluid Phase Equilibria, 283, 73-88 (2009).

3. R. T. JUBIN, "The Mass Transfer Dynamics of Gaseous Methyl-iodide Adsorption by Silver-exchanged Sodium Mordenite," Ph.D dissertation, The University of Tennessee (1994).

4. S. SIRCAR and J.R. HUFTON, "Why Does the Linear Driving Force Model for Adsorption Kinetics Work?” Adsorption, 6(2), 137 (2000).

5. Y.-S. HO, "Review of second-order models for adsorption systems," Journal of Hazardous Materials B136, 681-689 (2006).

6. H. QIU, L. LV, B. PAN, Q. ZHANG, W. ZHANG and Q. ZHANG, "Critical review in adsorption kinetic models," J Zhejiang Univ Sci A 10(5),716-724 (2009).

7. E. GLEUCKAUF and J. I. COATES, "Theory of Chromatography. Part IV. The Influence of Incomplete Equilibrium on the Front Boundary of Chromatograms and on the Effectiveness of Separation," J. Chem. Soc., 1315 (1947).

8. D. M. RUTHVEN, Principles of Adsorption and Adsorption Processes, pp. 141, 182, 242244, 1st Ed., Wiley-Interscience, New York, NY (1984).

9. W. E. RANZ and W. R. MARSHALL, "Evaporation from Drops," Chem. Eng. Progress, 48, 141 (1952).

10. N. WAKAO and T. FUNAZKRI, "Effect of fluid dispersion coefficients on particle-to-fluid mass transfer coefficients in packed beds: Correlation of sherwood numbers," Chemical Engineering Science 33(10), 1375-1384 (1978)

11. B. E. POLING, J. M. PRAUSNITZ and J. P. O'CONNELL, The Properties of Gases and Liquids, 5th Ed., McGraw-Hill, New York, NY (2000). 